\section{LA MONDIALISATION, VECTEUR DE LA CIRCULATION DES SOLUTIONS JURIDIQUES *}

\author{
LA GLOBALIZACIÓN, VECTOR DE LA CIRCULACIÓN \\ DE SOLUCIONES JURÍDICAS
}

A GLOBALIZAÇÃO, VETOR DA CIRCULAÇÃO DE
SOLUÇÕES JURIDICAS

\title{
RESUMEN
}

L'objectif de cet article est, après effectuer une réflexion sur les phénomènes de globalisation et mondialisation du droit, voir la façon dont le droit comparé apparaît comme une méthode essentielle pour les juges dans ce nouveau contexte. La conséquence principale est le développement de l'écoulement des solutions juridiques entre ces juridictions constitutionnelles grâce à l'utilisation du droit comparé. La question du droit de vote des prisonniers est utilisée comme un exemple pour illustrer ce phénomène d'interaction croissée.

\section{MOTS-CLEFS}

Circulation des solutions juridiques, droit comparé, globalisation, interprétation, juridiction constitutionnelle.

\footnotetext{
* Article de réflexion sur la globalisation du droit et son influence sur la circulation des solutions juridiques entre les juridictions constitutionnelles qui s'inscrit dans la continuité du travail doctoral de l'auteur sur le recours à la comparaison juridique par les juges constitutionnels.

a. Docteur en droit de l'université d'Aix-Marseille (France). Maître de conférences à la Faculté de droit de l'Université de Toulon (DICE CNRS-UMR 7318). Spécialiste de droit comparé et de droit constitutionnel. Membre de la Société de Législation Comparée et de la section française de l'Institut Ibérico-américain de droit constitutionnel.
}

Alexis Le Quinio ${ }^{a}$ lequinio@univ-tIn.fr Fecha de recepción: 26 de mayo de 2016 Fecha de revisión: 19 de octubre de 2016 Fecha de aceptación: 25 de octubre de 2016
MISIÓN JURÍDICA

Revista de Derecho y Ciencias Sociales

Bogotá, D.C. (Colombia)

Colaboradores Externos Internacionales

Núm. 12 Año 2017

Enero - Junio, pp. 47-67

ISSN 1794-600X 


\section{RESUMEN}

El objetivo de este artículo es, después de realizar una reflexión sobre los fenómenos de globalización, y globalización del derecho, mostrar cómo el derecho comparado aparece como un método esencial para los jueces en este nuevo contexto. La principal consecuencia es el desarrollo del flujo de las soluciones jurídicas entre los tribunales constitucionales a través del uso del derecho comparado. El tema del derecho de voto de los presos se utiliza como un ejemplo para ilustrar este fenómeno de interacción cruzada.

\section{PALABRAS CLAVES}

Circulación de las soluciones jurídica, derecho comparado, globalización, interpretación, jurisdicción constitucional.

\section{ABSTRACT}

The goal of this article is, after a reflection on the phenomenon of globalization and globalization of law to show how comparative law appears like an essential method for judges in this new context. The main consequence is the developpement of the circulation of judicial decisions between constitutional courts through the use of comparative law. The question of voting rights of prisoners is used as an example to illustrate this phenomenon of cross interaction.

\section{KEY WORDS}

Circulation of judicial solutions, comparative law, globalization, interpretation, constitutional court.

\section{RESUMO}

0 objetivo deste artigo é, após a realização de uma reflexão sobre os fenômenos da globalização, e globalização do direito, mostrar como o direito comparado aparece como um método essencial para os juízes neste novo contexto. A principal consequência é o desenvolvimento do fluxo das soluções jurídicas entre esses tribunais constitucionais através da utilização do direito comparado. A questão dos direitos de voto para os presos é usada como um exemplo para ilustrar este fenômeno crosstalk

\section{PALAVRAS-CHAVE}

Circulação de soluções legais, direito comparado, globalização, interpretação, jurisdição constitucional.
« Avec le progrès de la globalisation, les pratiques d'emprunt et de mimétisme institutionnel prennent une nouvelle dimension. Les processus de production des référentiels s'internationalisent, la lutte des Etats pour imposer leur propre vision du monde devient plus cruciale. Le développement des comparaisons internationales n'est pas étranger à cette recherche d'hégémonie » ${ }^{1}$.

\section{INTRODUCTION}

Une discipline scientifique peut connaître une évolution radicale lorsqu'intervient une révolution qui touche la matière dans ses fondements mêmes. Concernant la science juridique, plusieurs phénomènes ont concouru à sa mutation au cours du siècle précédent ${ }^{2}$. Le dernier, et non le moindre, est la mondialisation ${ }^{3}$, qui pourrait constituer la « vraie rupture du XX ${ }^{\text {ème }}$ siècle $»^{4}$.

Le droit lui-même est remis en cause par ce phénomène ${ }^{5}$, même si les juristes ont tardé à prendre le tournant global des sciences sociales ${ }^{6}$. Le droit n'est pas absolu ; bien au contraire, il est relatif dans l'espace. Il est avant tout national et son appréhension et sa définition passent par la délimitation de son champ d'application géographique. Il est cependant soumis à des influences ou des tendances étrangères, aujourd'hui plus que jamais.

1. B. JOBERT, « Politique de la comparaison », in LALLEMENT M., SPURKJ., Stratégies de la comparaison internationale, Paris, éd. du C.N.R.S., 2003, p. 325.

2. Parmi les phénomènes (dont certains sont connexes à la mondialisation) qui ont concouru à l'évolution du droit au 20 ème siècle, il convient de mentionner notamment le développement du droit international, la progression de l'Etat de droit, la généralisation de la justice constitutionnelle dans les Etats démocratiques ou encore la juridicisation de la société.

3.Concernant l'utilisation des vocables " mondialisation " et " globalisation ", voir infra. Dans le cadre de la présente étude, les deux termes seront utilisés de manière équivalente, car révélateurs d'une influence commune, indépendamment de toute considération lexicale.

4. P. JACQUET, P. SACHWALD, "Mondialisation : la vraie rupture du 20ème siècle », Politique étrangère, $n^{\circ} 3-4,2000$, pp. 597-612.

5. D. TURPIN, "Mondialisation et normes juridiques (Pour un nouveau contrat social global) ", in L'esprit des institutions, l'équilibre des pouvoirs. Mélanges en l'honneur de Pierre Pactet, Paris, Dalloz, 2003, p. 437.

6. A. CAILLE, S. DUFOIX, (dir.), Le tournant global des sciences sociales, Paris, éd. La Découverte, 2013, 440 p. 
La mondialisation, associée à l'interconnectivité normative, invite à repenser les bases conceptuelles et catégoriques de la science du droit et à adapter les modèles classiques à ces nouvelles données. Les ordres juridiques nationaux ont connu une révolution copernicienne sous l'influence de la mondialisation et de l'un de ses corollaires, la régionalisation.

Cette évolution affecte notamment les juridictions constitutionnelles qui, de par l'environnement juridique international actuel, sont poussées vers une coopération de plus en plus importante avec les autres juridictions, notamment supranationales. Il n'est pas aisé d'aborder la question de la globalisation dans l'optique du droit en général et du droit constitutionnel en particulier, la notion de Constitution étant traditionnellement liée à celle d'Etat-nation.

Le droit constitutionnel ne semble pas être influencé de manière significative par la mondialisation ; en effet, c'est le droit international qui en est le principal vecteur. Comme le rappelle le Professeur Cosnard, « l'instrument a priori idéal pour la diffusion des normes à l'échelle planétaire semble être le droit international» ${ }^{7}$. Toutefois, la mondialisation ne peut rester sans incidence sur le fonctionnement interne de l'Etat et sur le droit national. En l'occurrence, la question n'est pas de savoir comment la globalisation s'impose au droit constitutionnel mais comment le droit constitutionnel et ses juges s'adaptent à la globalisation ${ }^{8}$.

Le monde a changé, les sociétés aussi. Les Etats sont mis en concurrence dans de nombreux

7. M. COSNARD, «La création normative des Etats - Point de vue publiciste », in La mondialisation du droit, Colloque des 13, 14 et 15 sept. 1999 de Dijon, LOQUIN E., KESSEDJIAN C. (dir.), Travaux du centre de recherche sur le droit des marchés et des investissements internationaux, vol. 19, Paris, Litec, 2000, p. 150.

8. Il convient de distinguer ces problématiques de celles relatives à ce qui est qualifié, souvent de manière hâtive, de droit constitutionnel global. Sur cette question, voir J.-J. SUEUR, « Droit constitutionnel global ou droit global des constitutions? Eléments d'analyse », in A. LE QUINIO, (dir.), Les réactions constitutionnelles à la globalisation, Bruxelles, Bruylant, coll. A la croisée des droits, 2016, pp. 17-52; G. TUSSEAU, «Un chaos conceptuel qui fait sens : la rhétorique du constitutionnalisme global », in J.-Y. CHEROT, B. FRYDMAN (dir.), La science du droit dans la globalisation Bruxelles, Bruylant, 2012, pp. 182-227. domaines : politiques économiques et fiscales, protection sociale, systèmes éducatifs, innovation, etc. Les systèmes juridiques se retrouvent également en compétition. La demande de justice s'est accrue, affectant d'autant l'office du juge. Le temps actuel serait celui d'une "montée en puissance des juges ${ }^{9} »$ qui découlerait d'une judiciarisation et d'une juridicisation de la société accompagnée de la consécration d'une autonomisation sans cesse plus importante ${ }^{10}$.

Avec la mondialisation, les échanges entre les systèmes juridiques se sont multipliés, ouvrant la voie à un recours plus fréquent au droit comparé. Les juges nationaux ne sont plus isolés et interagissent avec leurs homologues. Avec le décloisonnement territorial qui marque leur travail, les juges deviennent plus autonomes par rapport à leur Etat, favorisant ainsi les critiques relatives à leur légitimité. Mais les juridictions « doivent [...] reconnaître mutuellement la valeur de leur jurisprudence respective, pour que le système reste globalement cohérent, quand bien même il n'y a pas de hiérarchie formelle entre ordres juridiques juxtaposés ${ }^{11} »$. Dans le cadre de ce nouvel environnement juridique international, les juges constitutionnels, par leur positionnement particulier et la visibilité de leur jurisprudence deviennent des acteurs de premier plan de la dynamique juridique globale.

La mondialisation n'oblige pas les juges à prendre en compte les exemples étrangers. Ils ne sont pas liés ${ }^{12}$, mais ils ne peuvent plus feindre de

9. M. DELMAS-MARTY, "Mondialisation et montée en puissance des juges ", in Le dialogue des juges, Actes du colloque organisé le 28 avril 2006 à l'Université libre de Bruxelles, Bruxelles, Bruylant, coll. Les Cahiers de l'Institut d'études sur la Justice, vol. 9, 2007, pp. 94-114.

10. J. ALLARD, A. VAN WAYENEBERGE, « De la bouche à l'oreille? Quelques réflexions autour du dialogue des juges et de la montée en puissance de la fonction de juger », R.I.E.J., vol. 61, 2008, pp. 112-113. Cette autonomisation serait marquée à la fois par l'émancipation des juges vis-à-vis des autres pouvoirs constitués, mais également dans son rapport à l'Etat puisque ce dernier n'est plus la seule source du droit positif applicable dans un système juridique donné.

11. K. LENAERTS, "Table ronde », ", in Le dialogue des juges, Actes du colloque organisé le 28 avril 2006 à l'Université libre de Bruxelles, Bruxelles, Bruylant, coll. Les Cahiers de l'Institut d'études sur la Justice, vol. 9, 2007, p. 124

12. Si certaines juridictions constitutionnelles intègrent les problématiques induites par la globalisation, il n'y a pas d'obligations pour ces juridictions de réagir à la globalisation. Sur cette question, voir A. LE QUINIO, (dir.), Les réactions constitutionnelles à la globalisation, Bruxelles, Bruylant, coll. A la croisée des droits, 2016, 351 p. 
ne pas connaître les solutions proposées par leurs collègues issus d'autres systèmes juridiques. C'est le statut même de l'information juridique qui a été modifié par son accessibilité. Le développement du marché par la globalisation a également touché le monde des juristes qui sont davantage soumis à une logique concurrentielle.

Sous l'effet de la globalisation du droit, les juges constitutionnels sont amenés à s'ouvrir davantage aux influences externes et à participer à la migration des solutions juridiques d'un système juridique à l'autre.

\section{De la globalisation}

Le vocable « mondialisation ${ }^{13}$, avant tout utilisé comme qualificatif, est aujourd'hui répandu dans l'ensemble de la presse généraliste et spécialisée. Il est le plus souvent employé pour qualifier un phénomène de globalisation qui concerne l'ensemble des activités économiques via notamment, une accentuation des échanges et des flux dans le cadre d'une intégration économique généralisée ${ }^{14}$. En effet, c'est dans la science économique que la notion a trouvé son origine. La réduction des distances, la perméabilité des frontières introduites par la mondialisation ont eu davantage de conséquences dans la perspective d'un marché mondial que dans celle d'une justice globale.

La mondialisation est appréhendée habituellement selon deux volets distincts. Le premier est de l'ordre du discours et constitue une modalité de gestion du monde « postmoderne $»^{15}$. Cette idéologie repose sur quelques croyances simples liées aux bienfaits supposés des lois du marché qui, via le libéralisme ${ }^{16}$, l'ouverture des

13. L'utilisation, dans la littérature française et francophone du vocable "mondialisation » en lieu et place de celui de " globalisation ", privilégié dans les travaux anglo-saxons et allemands, est souvent critiquée, bien que du point de vue de l'étymologie et de la connotation, il semble que le caractère « mondial » du phénomène soit plus précis, voir BOMPARD-PORTE M., "Questions de mots ", in Images de la mondialisation - La construction sociale d'une représentation, Actes du colloque international «Représentations de la Mondialisation », tenu à Brest les 17 et 18 nov. 2005, Rennes, P.U.R., coll. Des Sociétés, 2008, pp. 17-22.

14. C.-A. MICHALET, "Les métamorphoses de la mondialisation, une approché économique ", in La mondialisation du droit, Colloque des 13, 14 et 15 sept. 1999 de Dijon, LOQUIN E., KESSEDIIAN C. (dir.), Travaux du centre de recherche sur le droit des marchés et des investissements internationaux, vol. 19, Paris, Litec, 2000, pp. 11-42. frontières et la libre-concurrence, seraient les garants de la croissance et du progrès ${ }^{17}$. Le second, celui qui nous intéresse, est un concept caractérisant l'apparition d'un processus ou plutôt d'une série de processus qui ne sont pas affectés par les frontières ou les autres barrières institutionnelles et qui favorisent certaines pratiques dans les domaines économique, politique, culturel, social, etc.

Telle qu'elle est généralement présentée, la mondialisation laisse à penser qu'elle est une force immanente qui s'impose à l'ensemble de la planète, expliquant en partie pourquoi elle « n'a pas bonne réputation ${ }^{18}$. Ce postulat ne tient pas compte de la dynamique interne de la mondialisation. Ce sont les gouvernants qui ont fait le choix de cette intégration sans cesse plus étroite, d'abord dans le domaine économique puis dans les différents champs de la vie sociale. Alors qu'elle se limitait initialement aux pays dits " développés », la mondialisation s'est ensuite étendue aux pays en voie de développement qui ont considéré ce processus comme une opportunité d'attirer des capitaux et des technologies.

Cette croissance des flux internationaux n'a été possible que grâce au développement technologique et à l'innovation dans les transports ainsi que dans les nouvelles technologies de l'information et de la communication. On observe une diminution généralisée de ce que les économistes appellent " les coûts de transaction ${ }^{19}$, rendant ainsi plus aisé l'accès aux informations pertinentes pour les différents acteurs de l'économie, particulièrement

15. Sur la notion de postmodernisme en droit, voir A.-J. ARNAUD Entre modernité et mondialisation - Leçons d'histoire de la philosophie du droit et de l'Etat, 2ème éd., Paris, L.G.D.J., Droit et société, Série Droit, $n^{\circ} 20,2004, p p$. 265-299. J. CHEVALLIER L'Etat postmoderne, Paris, L.G.D.J., 2001, 225 p. "Vers un droit post-moderne? Les transformations de la régulation juridique, R.D.P., $n^{\circ} 3,1998$, pp. 659-714

16. Pour une relativisation du lien entre le libéralisme et le développement économique, voir G. FEUER, "Libéralisme, mondialisation et développement. A propos de quelques réalités ambigües », A.F.D.I., vol. 45, 1999, pp. 148-164.

17. R. L. KUTTNER, "Development, Globalization, and Law », Mich. J. Int'l L., $n^{\circ} 26,2004, p p$. 19-38.

18. D. MOCKLE, «Mondialisation, droit des peuples et Etat de droit ", in MOCKLE D. (dir.), Mondialisation et Etat de droit, Bruxelles, Bruylant, coll. Mondialisation et droit international, 2002, p. 1.

19. Voir les travaux d'O. E. WILLIAMSON pour caractériser les coûts induits par le recours au marché pour effectuer l'échange. 
les multinationales, acteurs privilégiés de cette nouvelle géographie économique.

Une des manifestations de cette problématique est la mise en place d'institutions dédiées. En effet, de nombreuses entités juridiques supranationales ont été créées dans la seconde moitié du XXème siècle. Elles sont constituées généralement par un ensemble de dispositions contraignantes et une juridiction chargée d'assurer la cohérence et l'efficacité du mécanisme. C'est dans le domaine économique que cette approche institutionnelle a d'abord vu le jour. Au niveau global, on retrouve le General Agreement on Tarifs and Trade auquel a succédé l'Organisation mondiale du commerce pour une diminution et une harmonisation des droits de douanes, le Fond monétaire international, dont l'objectif est la promotion de la coopération monétaire et de la stabilité financière. Au niveau régional, le processus s'est développé par la création de marchés communs tels que la Communauté économique européenne, devenue plus tard l'Union européenne, le North American Free Trade Agreement ou le Mercado Común del Sur.

Une des évolutions les plus significatives est la mobilité particulièrement importante des données ${ }^{20}$. Ce phénomène a entraîné un changement qualitatif et quantitatif de la pensée, bouleversant les méthodes d'investigation et d'appréhension d'un élément. Nous observons une densification du tissu d'informations, la difficulté ne résidant plus dans l'acquisition des données, mais dans leur sélection. Au-delà de la globalisation financière, le développement d'Internet a engendré les conditions d'une libre circulation des flux d'informations ${ }^{21}$. Les différents acteurs de ce nouvel ordre mondial doivent avant tout privilégier l'évaluation de l'information ; la perspective doit être qualitative.

La mondialisation s'inscrit ainsi dans un mouvement qui modifie en profondeur notre " lecture » du monde. La globalisation est multidimensionnelle. Elle marque une

20. J. BASEDOW, «Vie universelle. Droit national ? A propos de la mondialisation du droit ", in Mélanges en l'honneur de Denis Tallon - D'ici, d'ailleurs : harmonisation et dynamique du droit, Paris, SLC, 1999, p. 226.

21. La science du droit est également bouleversée par l'accessibilité des données. La multiplication des sites communautaires et des sites d'informations, ainsi que la création de revues en ligne et de bases de données à vocation scientifique ont conduit les chercheurs à repenser leur méthode de travail. multiplication des échanges de biens et de services consécutive à la diminution des barrières protectionnistes, une augmentation de la puissance des acteurs économiques transnationaux et la prédominance de la logique financière de l'économie via la spéculation. Son influence ne se limite toutefois pas au domaine économique et affecte également la sphère juridique.

\section{La globalisation du droit}

La mondialisation est multiple, tant dans les champs dans lesquels on l'observe, que dans la manière dont elle s'insère dans ces derniers. Dans le domaine juridique, elle est principalement double ${ }^{22}$. A une dimension fonctionnelle évidente de mise en rapport et de résolution de conflits entre différentes sphères d'échanges, est apparue, concomitamment, une forte volonté éthique inscrite dans une dimension humaniste et « universalisante ».

$\mathrm{Au}$ niveau symbolique, la mondialisation s'identifie en effet à la généralisation de l'économie de marché et à l'adhésion aux valeurs démocratiques liées à une certaine idée des droits de l'homme. Pour Mireille Delmas-Marty, la mondialisation est un terme neutre, la globalisation concerne l'économie et l'universalisation les droits de l'homme ${ }^{23}$. D'après elle, donner une autre signification à la mondialisation est une résignation trop rapide au « primat de l'économie sur celui des droits de l'homme $»^{24}$.

La mondialisation se manifeste avant tout par la mise en place graduelle d'un marché à l'échelle planétaire. La mondialisation, par son influence, ne favorise pas tant un «droit commun » qu'un « droit global » qui se concrétise dans

22. . ALLARD, A. GARAPON, Les juges dans la mondialisation La nouvelle révolution du droit, Paris, éd. du Seuil, coll. La République des Idées, 2005, p. 6.

23. La construction européenne semble caractéristique de cette approche. Elle a commencé avec une Europe du marché, une Communauté économique européenne et une Europe des droits de l'Homme dans le cadre du Conseil de l'Europe. Le cloisonnement de ces deux entités a rapidement montré ses limites. Cette coexistence, cet enchevêtrement, sur un même territoire, a conduit les Etats à repenser l'organisation institutionnelle de l'Union et à la faire évoluer.

24. M. DELMAS-MARTY, Trois défis pour un droit mondial, Paris, éd. du Seuil, coll. Essais, 1998, pp. 1415. 
la perspective du marché par une primauté de la concurrence. Ce marché se caractérise par la mobilité des biens et des services, des capitaux, des facteurs productifs et même des hommes. Les libertés de circulation revêtent en l'espèce une importance particulière. Elles constituent le fondement de l'intégration économique et institutionnelle, à l'image de leur influence dans la perspective de la construction européenne.

La mondialisation du droit s'est développée dans un premier temps dans des disciplines techniques. Selon Hélène Blanc, « les relations économiques internationales sont généralement présentées comme une dimension prégnante, voire le vecteur essentiel, de la mondialisation contemporaine $»^{25}$. De la même façon que l'Union européenne s'est construite sur le fondement de la coopération économique avant de s'étendre à tous les domaines de la vie sociale, la mondialisation, au même titre que l'harmonisation, " a plus de chance de réussite quand elle aborde des sujets émotionnellement peu chargés, comme le transport ou le commerce $»^{26}$. Cette perception de la mondialisation, comme uniquement tournée vers l'efficacité économique, parfois au mépris d'autres impératifs, explique en grande partie le caractère social de sa contestation.

Ainsi, les effets les plus visibles de la mondialisation se sont fait sentir en droit des conventions, en droit du travail ou en droit des finances. Mais il ne faut pas pour autant sousestimer l'impact du phénomène sur les autres branches du droit. Le droit pénal, pourtant davantage marqué par le lien sociétal et la culture juridique du pays dans lequel il est né, a été l'un des premiers touchés par la globalisation du droit ${ }^{27}$.

25. H. BLANC, «De l'internationalisation des économies à leur mondialisation, le regard de l'économiste ", in Images de la mondialisation - La construction sociale d'une représentation, Actes du colloque international "Représentations de la Mondialisation", tenu à Brest les 17 et 18 nov. 2005, Rennes, P.U.R., coll. Des Sociétés, 2008, p. 39.

26. H. VAN HOUTTE, "La modélisation substantielle », in LOQUIN E., KESSEDIIAN C. (dir.), La mondialisation du droit, Colloque des 13, 14 et 15 sept. 1999 de Dijon, Litec, Travaux du centre de recherche sur le droit des marchés et des investissements internationaux, vol. 19, 2000, pp. 207-236.

27. M. DELMAS-MARTY, « La pénalisation internationale des activités économiques : un espace à géographie variable ", in LOQUIN E., KESSEDJIAN C. (dir.), La mondialisation du droit, Colloque des 13, 14 et 15 sept. 1999 de Dijon, Paris, Litec Travaux du centre de recherche sur le droit des marchés et des investissements internationaux, vol. 19, 2000, pp. 401-414.
Il importe toutefois de relativiser l'impact de la mondialisation du droit sur la dynamique juridique prise dans son ensemble. Le rôle des systèmes juridiques nationaux est encore primordial. Les thèses défendues par André-Jean Arnaud du dépassement de l'Etat moniste ${ }^{28}$ par le droit global et le pluralisme nous paraissent diminuer de manière excessive le rôle joué, encore aujourd'hui, par les ordres juridiques nationaux. Le droit international est majoritairement interétatique et le droit global lui-même, provient en grande partie des Etats $^{29}$. Il est nécessaire de ne pas exagérer l'influence de la globalisation, ni verser dans le propos journalistique à propos de termes ( " global » ou «mondial ») trop souvent utilisés et trop peu souvent définis mais il convient d'intégrer les modifications apportées par ce processus au paradigme traditionnel d'étude du droit ${ }^{30}$.

Si la globalisation est un phénomène à la portée indéniable, il faut en délimiter correctement le champ d'action. La multiplication des échanges juridiques et le développement de nouveaux systèmes juridiques qui en découlent jouent essentiellement au niveau « infra-global $»^{31}$. La communauté des juristes est confrontée à une mutation profonde, à une complexification de son environnement : " As time moves on, and commerce, telecommunications, culture and transport become increasingly globalized, a growing body of law and norms will emerge that is, on the one hand, universally recognized, but on the other, neither wholly domestic nor wholly international in its character or origin $»^{32}$.

La compréhension du phénomène de globalisation du droit nécessite une approche structurée qui permettrait de synthétiser de

28. A.-J. ARNAUD, Entre modernité et mondialisation - Leçons d'histoire de la philosophie du droit et de l'Etat, Paris, L.G.D.J., coll. Droit et société, Série Droit, vol. 20, 1998, pp. 160-161.

29. P. BIRNBAUM, "Le type d'Etat tient toujours ", in L'architecture du droit, mélanges en l'honneur de Michel Troper, Paris, Economica, 2006, pp. 185-194.

30. Le Président ZAGREBELSKY qualifie les transformations récentes du droit et de l'Etat de " mutation génétique ", in Le droit en douceur, Paris - Aix-en-Provence, Economica - P.U.A.M. Coll. Droit Public Positif, 2000, pp. 7-8.

31. D. A. WESTBROOK, «Keynote Adress : Theorizing the Diffusion of Law : Conceptual Difficulties, Unstable Imaginations, and the Effort to Think Gracefully Nonetheless ", Harv. Int'l L. J., vol. 47, 2006, pp. 493-496.

32. $\mathrm{KOH} \mathrm{H.} \mathrm{H.,,} \mathrm{"The} \mathrm{Globalization} \mathrm{of} \mathrm{Freedom} \mathrm{»,} \mathrm{Yale} \mathrm{J.} \mathrm{Int'l} \mathrm{L.,}$ vol. 26, 2001, p. 306. 
manière didactique les interactions entre systèmes juridiques. Pour Guy Canivet, c'est par le prisme de la culture juridique que cette pensée doit prospérer ${ }^{33}$.

La culture juridique peut être définie comme un modèle de comportement social et d'attitudes juridiquement orienté ${ }^{34}$. C'est un ensemble de techniques d'exposition et d'interprétation employé par les opérateurs économiques, ainsi que l'idéologie, les valeurs et principes qu'elles véhiculent ${ }^{35}$. D'après Patrick Glenn, la notion de « tradition juridique » serait plus pertinente, en ce qu'elle prendrait davantage en compte certains éléments historiques ainsi que la possibilité de lui voir un effet normatif ${ }^{36}$.

Que ce soit par le prisme de la tradition ou de la culture juridique, les systèmes juridiques sont soumis à un enchevêtrement normatif de plus en plus dense, spécialement lorsqu'ils appartiennent à des ensembles juridiques supranationaux. Ces enchevêtrements entraînent l'apparition d'un phénomène de rapprochement qui s'opère essentiellement entre des pays placés dans des situations de développement équivalentes au sein d'aires géographiques communes.

Dans cette perspective, la mondialisation ne doit pas être perçue comme équivalente à l'internationalisation. Si cette expression est plus familière aux juristes, elle recouvre une fonction différente, l'internationalisation ne constituant que le stade préliminaire à la première ${ }^{37}$. Si

33. G. CANIVET, "La convergence des systèmes juridiques par l'action du juge ", in De tous horizons, Mélanges Xavier BlancJouvan, Paris, S.L.C., 2005, p. 12.

34. D. NELKEN, "Using the Concept of Legal Culture », conférence prononcée devant l'association australienne de philosophie juridique et sociale en juillet 2003, accessible sur le site de l'Université de Californie à l'adresse : http://www.law.berkeley. edu/institutes/csls/nelken\%20paper.pdf

35. G. REBUFFA, "Culture juridique », in ARNAUD A.-J. (dir.), Dictionnaire encyclopédique de théorie et de sociologie du droit, 2ème éd., Paris, LGDJ, 1993, pp. 139-141.

36. P. H. GLENN, "La tradition juridique nationale », R.I.D.C., vol. $55, n^{\circ} 2$, pp. 263-278. Pour une approche globale de la question comme prisme d'analyse du droit, voir P. H. GLENN, Legal Traditions of the World - Sustainable Diversity in Law, 3ème éd., Oxford, Oxford University Press, 2007, 395 p.

37. Pour René-Jean DUPUY, " l'internationalisation du Droit doit compter avec un phénomène considérable qui exerce sur elle tout à la fois un effet démultiplicateur et un effet réducteur : la mondialisation de l'économie », voir "introduction ", in L'internationalité dans les institutions et le droit, Etudes offertes à Alain Plantey, Paris, Pedone, 1995, p. XXII. l'internationalisation maintient au centre de l'activité juridique la nation, dans son interaction avec les autres, la globalisation, elle, remet en cause ces rapports ${ }^{38}$. Tandis que la première prend appui sur la volonté étatique, la seconde échappe au contrôle des Etats ${ }^{39}$. En effet, alors que l'adoption de certains amendements à des lois nationales s'apparente à un chemin de croix politique et institutionnel, la ratification de textes internationaux et l'intégration dans l'ordre interne de pans entiers de législations semble poser moins de difficultés.

L'internationalisation repose sur une volonté de mise en commun tandis que la globalisation repose sur une nécessité. Elle est la conséquence du fait que certains problèmes ne peuvent plus être appréhendés de manière autre que globale, à une échelle mondiale. Ces sujets " globaux " de préoccupation sont légion : l'environnement, la sécurité alimentaire, l'accès à l'eau, les droits de l'homme, la protection de la faune, la stabilité financière, la lutte contre le terrorisme ou les inégalités, etc. Ces nouveaux domaines, au même titre que les questions de biotechnologie ou de gestion des produits financiers, ne peuvent plus être efficacement encadrés par des normes uniquement nationales. Si certains de ces thèmes sont liés à la question du commerce, ils ne peuvent être réduits à cette seule dimension. La mondialisation du droit n'est pas synonyme d'internationalisation du droit, et si c'était le cas, elle ne constituerait alors qu'une extension du champ matériel du droit international.

Le professeur Chevalier le confirme : « la mondialisation correspond en effet à une étape radicalement différente de l'internationalisation qui s'était développée après la Seconde Guerre mondiale : alors que l'internationalisation prenait appui sur les Etats-Nations, qui continuaient à s'imposer comme des dispositifs nécessaires de médiation, la mondialisation échappe largement à leur emprise ; et sa portée n'est pas seulement économique, mais

38. A.-J ARNAUD., Entre modernité et mondialisation - Leçons d'histoire de la philosophie du droit et de l'Etat, 2ème éd., Paris, L.G.D.J., Droit et société, Série Droit, $n^{\circ} 20,2004$, p. 22.

39. Cette évolution de la perspective n'est pas propre à la science juridique et touche l'ensemble des sciences humaines voir P. HASSENTEUFEL, «De la comparaison internationale à la comparaison transnationale », R.F.S.P., 2005, pp. 113-132. 
aussi sociale, culturelle, politique $»^{40}$. L'une des principales caractéristiques du phénomène est donc son extension à l'ensemble des activités sociales et humaines, même si elle les affecte avec une intensité différente.

La mondialisation du droit emporte
des conséquences plus profondes que l'internationalisation. En effet, cette dernière est limitée par le caractère embryonnaire de la justice internationale notamment du fait que la séparation des pouvoirs est difficilement transposable dans l'ordre des relations interétatiques ${ }^{41}$. Quant à l'expression " universalisation du droit » qui est parfois utilisée, son implication idéologique est plus fortement marquée. Elle est utilisée comme une réinterprétation du processus de mondialisation dans la perspective d'un "partage de sens » qui dépasserait la simple diffusion spatiale, et dont les droits de l'homme seraient le vecteur privilégié ${ }^{42}$.

Certes, la dimension du phénomène le rend incontournable, mais c'est bien sa nouveauté qui marque avant tout. La mondialisation a pénétré l'ensemble des champs de la recherche, mais ce n'est que récemment que les juristes s'en sont saisis. L'interaction normative et la circulation des objets juridiques constituent une véritable nouveauté, remplaçant les traditionnelles migrations juridiques. Ce n'est pas une simple question de vocabulaire, où le terme " mondial " remplacerait celui d' " international » pour qualifier une dynamique différente. Les processus d'internationalisation, d'européanisation et de globalisation, s'ils ne sont pas similaires, " se conjuguent de manière à rendre les systèmes juridiques nationaux plus perméables aux règles de droit international et de droit étranger $»^{43}$.

40. J. CHEVALLIER, "Mondialisation du droit ou droit de la mondialisation ?", in C.-A. MORAND, (dir.), Le droit saisi par la mondialisation, Bruylant, Coll. De droit international, Ed. De l'Université de Bruxelles, Bruxelles, Helbing \& Lichtenhahn Verlag, 2001, p. 37.

41. G. DE LA PRADELLE, "Juridicisation de la société et globalisation ", in CHEMILLIER-GENDREAU M., MOULIERBOUTANG Y., (dir.), Le droit dans la mondialisation - Une perspective critique, Paris, P.U.F., 2001, p. 25.

42. M. DELMAS-MARTY, Trois défis pour un droit mondial, Paris, éd. du Seuil, coll. Essais, 1998, pp. 14 15. L'auteur reprend à son compte en la précisant la distinction établie par Zaik Laidi.

43. M.-C. PONTHOREAU, "La concurrence entre Common Law et droit civil. Existe-t-il véritablement une concurrence entre Common Law et tradition civiliste ? Le point de vue du comparatiste de droit public ", in DU BOIS DE GAUDUSSON
Dans cette perspective, André-Jean Arnaud soutient la thèse selon laquelle ce phénomène, qui touche directement le droit, permet un renouvellement des problèmes auxquels cette discipline est généralement confrontée. Il affirme en effet que " débordant les matières économiques et financières, le terme "global" s'est imposé dans une acception paradigmatique au sens fort $\aleph^{44}$. Tandis que l'internationalisation se limite à un objet ou une activité déterminée, la globalisation a vocation à étendre le phénomène à l'ensemble des pans de l'activité humaine.

Beaucoup de préjugés subsistent dans l'approche du rapport entre droit et mondialisation. Tandis qu'elle était initialement perçue comme le corollaire nécessaire de l'harmonisation et de l'universalisation des droits, la globalisation du système international, notamment dans son lien avec la dynamique du marché, entraine des réactions négatives renforçant les revendications identitaires. Selon Jacques Chevallier, les rapports entre droit et mondialisation sont ambivalents ; " d'un côté, elle travaille les ordres juridiques [...] de l'autre, la mondialisation entraîne la production d'un corpus de règles juridiques spécifiques $»^{45}$. En somme, nous voyons apparaître un « droit mondialisé » et un droit de la mondialisation, puisque « non seulement le droit issu de la mondialisation ne conduit pas toujours à la mondialisation du droit interne, mais il peut aboutir à la nationalisation du droit mondial $»^{46}$.

Cette complexification et cette imbrication des systèmes juridiques sous l'effet de la globalisation nécessitent de nouveaux outils de réflexion et de nouvelles méthodes de travail afin

J., FERRAND F. (dir.), La concurrence des systèmes juridiques, Actes du colloque organisé le 20 octobre 2006 à l'Université Jean Moulin Lyon III, Aix-en-Provence, P.U.A.M., 2008, p. 37.

44. A.-J. ARNAUD, Entre modernité et mondialisation - Leçons d'histoire de la philosophie du droit et de l'Etat, 2ème éd., Paris, L.G.D.J., Droit et société, Série Droit, $n^{\circ} 20,2004$, p. 28.

45. J. CHEVALLIER, "Mondialisation du droit ou droit de la mondialisation ? ", in MORAND C.-A., (dir.), Le droit saisi par la mondialisation, Bruxelles, Bruylant, Editions de l'Université de Bruxelles, Collection de droit international, $n^{\circ} 46$, Helbing \& Lichtenhahn Verlag, 2001, p. 38.

46. M. DELMAS-MARTY, " Les processus de mondialisation du droit ", in MORAND C.-A., (dir.), Le droit saisi par la mondialisation, Bruxelles, Bruylant, Editions de l'Université de Bruxelles, Collection de droit international, $n^{\circ} 46$, Helbing \& Lichtenhahn Verlag, 2001, p. 68. 
de s'adapter à ces nouveaux enjeux. Dans cette optique, la méthode juridique comparative tend à jouer un rôle fondamental.

\section{Globalisation et droit comparé}

La globalisation a modifié notre façon de penser le droit et sa dynamique. Le développement du droit doit être appréhendé dans une perspective renouvelée, la globalisation nécessitant « a revival of general jurisprudence and a rethinking of comparative law from a global perspective as key elements in cosmopolitan legal studies ${ }^{47}$. Le décloisonnement des systèmes juridiques entraîné par la globalisation tend à renforcer le rôle accordé au droit comparé.

A titre principal, la mondialisation constitue un changement d'échelle, c'est-à-dire que les problématiques qui antérieurement se posaient au niveau des Etats concernent désormais des ensembles juridiques et des aires géographiques plus vastes, pouvant aller jusqu'à la planète entière. C'est ce constat qui met au premier plan le droit comparé. En effet, « la comparaison peut et doit devenir, pour une discipline que l'immobilisme conduirait doucement vers l'Histoire, l'un des facteurs essentiels des progrès et des renouvellements nécessaires à la vie $»^{48}$.

La principale conséquence de cette évolution n'est pas la consécration d'un nouvel ordre juridique global/mondial, mais bien la pénétration du phénomène de globalisation dans les référents traditionnels que constituent les systèmes juridiques nationaux.

L'élaboration du droit se " dénationalise », particulièrement dans le champ des droits de l'homme. Les interactions normatives se multiplient. La globalisation a consacré un « temps de légalité poreuse ou de porosité juridique, où de multiples réseaux d'ordres juridiques nous forcent constamment à des transitions ou des empiètements. Notre vie

47. W. TWINING, Globalization and Legal Theory, Evanston, Northwestern University Press, 2000, p. 189.

48. J. RIVERO, " Droit administratif français et droits administratifs étrangers ", in DE LAUBADERE A., MATHIOT A., RIVERO J., VEDEL G., Pages de doctrine, Paris, L.G.D.J., 1980, $t$. 2, p. 485 . juridique consiste en l'intersection de différents ordres juridiques, c'est-à-dire l'interlégalité ${ }^{49}$.

La mondialisation a bouleversé le monde des juristes. Ils ont été obligés de repenser en profondeur leurs habitudes ; ils ont dû se tourner vers l'extérieur, s'ouvrir. Contrairement à une idée répandue, la globalisation n'est pas dominée par l'influence nationale sur le droit et les procédures internationales, mais entraîne davantage une internationalisation des systèmes et des comportements judiciaires nationaux.

Pour François Ost et Michel van de Kerchove, chaque époque appelle une théorie du droit déterminée qui doit reposer sur la compréhension $\mathrm{du}$ contexte culturel de l'époque qui s'impose comme le préalable obligé à toute « intelligence du droit $»^{50}$. En l'espèce, nous pensons que cette intelligence doit aujourd'hui reposer sur la méthode juridique comparative qui est au cœur du travail de la communauté des juristes.

Le droit comparé constitue un moyen essentiel offert à cette communauté pour appréhender les mutations de son objet d'étude. Sous l'impact de la mondialisation et du métissage juridique qu'elle accentue, le droit comparé a vu son " déficit d'image $»^{51}$ remis en question. Il offre aux juristes un formidable outil de réflexion et de travail dans ce nouveau contexte.

Les difficultés à concevoir la mondialisation sont les mêmes que celles afférentes à la méthode juridique comparative ${ }^{52}$. Les instruments analytiques auxquels les juristes sont habitués sont insuffisants pour saisir ce phénomène dans sa globalité et dans ses potentialités. Comme le souligne le professeur William Twining : "We are not yet well-equipped to talk globally about law $»^{53}$. Néanmoins,

49. A.-J. ARNAUD, Pour une pensée juridique européenne, Paris, P.U.F., coll. Les voies du droit, 1991, p. 235.

50. F. OST, M. KERCHOVE (van de), Le droit ou les paradoxes du jeu, coll. Les voies du droit, Paris, P.U.F, 1992, p.7.

51. A. J. BULLIER, «Le droit comparé est-il un passe temps inutile ?», R.D.I.D.C., $n^{\circ} 2 \& 3,2008$, p. 164.

52. La question se pose de savoir s'il est possible de parler d'une méthode comparative proprement dite ou si elle se concrétise par l'intermédiaire d'autres méthodes: herméneutique, structurelle, fonctionnelle, etc. En toute hypothèse et sans rentrer dans le débat épistémologique, la méthode juridique comparative peut être identifiée comme un savoir-faire. 
nous ne sommes pas totalement démunis. La méthode juridique comparative, utilisée à bon escient, apparaît comme l'outil indispensable à la prise en compte de ce processus.

La perméabilité des systèmes juridiques nationaux « brings laws and legal cultures into more direct, frequent, intimate, and often complicated and stressed contact. It influences what legal professionals want and need to know about foreign law, how they transfer, acquire, and process information, and how decisions are made. We might expect the field of comparative law, therefore, to be replete with efforts to comprehend globalization and its impacts on law and to develop strategies for dealing with them $»^{54}$. Sous l'effet conjugué des divers bouleversements qui affectent les systèmes juridiques, de nombreuses problématiques classiques nécessitent d'être repensées, à l'image de la question de la séparation des pouvoirs ${ }^{55}$.

La mondialisation se caractérise avant tout par une « remodélisation » de l'espace. Les frontières, prises au sens strict mais également dans une acception plus large sont remises en cause. Nous ne sommes pas encore dans un village global, les espaces nationaux et régionaux sont toujours des acteurs essentiels, mais ils ne sont plus exclusifs. Alors que la mondialisation de l'économie est marquée par la déterritorialisation de la production, la globalisation juridique marque une dénationalisation ${ }^{56} \mathrm{du}$ droit et de son élaboration.

Pour les comparatistes, la mondialisation entraîne une multiplication des sources et des niveaux de comparaison, ainsi qu'une

53. W. TWINING, «Diffusion and Globalization Discourse », Harv. Int'l L. J., vol. 47, 2006, p. 510 .

54. D. J. GERBER, "Globalization and Legal Knowledge : Implications for Comparative Law », Tul. L. Rev., $n^{\circ}$ 75, 2001, $p$. 950.

55. F. OST et M. KERCHOVE (van de), "Le juge entre ordre et désordre ", in Dire le droit, faire justice, coll. Penser le droit, Bruxelles, Bruylant, 2007, p. 75. D'après ces auteurs, elle doit s'appréhender comme " une collaboration régulée par un esprit de réserve ou d'autolimitation ».

56. Pour le professeur Caillosse, cette dénationalisation constitue une réelle opportunité pour les juristes ; "la dénationalisation $d u$ droit ouvre de nouvelles perspectives intellectuelles », voir CAILLOSSE J., "Sur quelques problèmes actuels du droit administratif français. Bref essai de mise en perspective ", A.J.D.A., 2010, p. 936. complexification croissante de l'articulation entre ces différentes normes et ces différents niveaux. Cette problématique se pose notamment dans le rapport entre le système juridique national, étalon de notre appréhension du droit et ces nouvelles « sources » normatives. Mireille Delmas-Marty estime d'ailleurs que la globalisation entraîne un déplacement des enjeux du droit comparé, davantage orienté vers la pratique. Les méthodes doivent être axées « vers l'analyse des influences croisées entre systèmes et l'évaluation des solutions fournies par les différents systèmes dans tel ou tel domaine $»^{57}$.

Sous l'effet conjugué de ces différents processus de remise en cause du cadre national comme sphère d'étude (mondialisation, gouvernance, européanisation, etc.), la question du changement et du développement du droit se place au cœur de la recherche juridique.

La démarche comparative voit sa légitimité renforcée par les interactions de plus en plus étroites entre les systèmes juridiques nationaux, la facilité accrue d'accès aux informations juridiques relatives aux autres systèmes ainsi que le rôle d'intégration joué par les nouveaux ensembles supranationaux. Pour le professeur Gerber, la mondialisation exige le développement d'outils plus sophistiqués pour procéder à la comparaison et à l'interprétation des connaissances juridiques étrangères ainsi qu'une attention particulière aux processus $\mathrm{d}^{\prime}$ acquisition de l'information juridique ${ }^{58}$.

$\mathrm{Si}$ les droits fondamentaux constituent une terre d'élection privilégiée pour la mondialisation du droit, cela provient avant tout de leur caractère parfois « anational ». C'est le cas par exemple du droit à un environnement sain ; la question ne peut pas être traitée dans les limites d'un Etat, sous peine de diminuer la portée des efforts fournis.

Le rapport au temps a également été largement modifié. La globalisation a réduit les distances et le temps. Grâce aux nouvelles technologies de l'information et au progrès

57. M. DELMAS-MARTY, Critique de l'intégration normative, Paris, PUF, coll. Les voies du droit, 2004, p. 20.

58. D. J. GERBER, « Globalization and Legal Knowledge: Implications for Comparative Law», Tul. L. Rev., $n^{\circ}$ 75, 2001, p. 950. 
des réseaux de communication, les acteurs juridiques ont dû développer une approche fondée sur l'instantané et le simultané.

Le juge est l'acteur juridique le plus susceptible de s'adapter à la nouvelle temporalité du droit. Ainsi, le droit jurisprudentiel peut constituer un " instrument de référence privilégié pour consacrer une mondialisation du droit, qui serait non pas une mondialisation/ uniformisation, mais davantage une mondialisation/harmonisation $»^{59}$.

L'apparition de ces nouvelles problématiques, couplée à la globalisation, conduit à considérer le droit comparé comme le moyen de saisir l'interdépendance des ordres juridiques. Le juge en a pris conscience et participe aux mutations qui affectent son travail. La justice s'est ouverte et internationalisée. Les juges, particulièrement ceux chargés du contrôle de constitutionnalité des lois et de la protection des droits fondamentaux, ont été sensibilisés au travail de leurs homologues ${ }^{60}$, notamment par l'intermédiaire d'initiatives associatives. Les juges, sous l'influence de la mondialisation et de l'interpénétration des espaces normatifs, sont davantage confrontés au droit comparé. Les échanges entre eux se multiplient et favorisent une plus grande circulation des solutions juridiques.

\section{La circulation des solutions juridiques}

La globalisation, de par son influence, en favorisant notamment le recours à la méthode juridique comparative - qui devient un élément de compréhension et de travail incontournable pour les différents acteurs juridiques - sert d'accélérateur à la migration des idées constitutionnelles.

59. M. COSNARD, "La création normative des Etats - Point de vue publiciste ", in La mondialisation du droit, Colloque des 13, 14 et 15 sept. 1999 de Dijon, LOQUIN E., KESSEDJIAN C. (dir.), Travaux du centre de recherche sur le droit des marchés et des investissements internationaux, vol. 19, Paris, Litec, 2000, p. 158.

60. R. ERRERA, "The Use of Comparative Law Before the French Administrative Law Courts », in CANIVET G., ANDENAS M., FAIRGRIEVE D. (éd.), Comparative Law before the Courts, British institute of International and Comparative Law, Londres, 2004, p. 162 : "Inside the EU today the words "cross-fertilization", "harmonisation of legislation", and "ius commune" are widely used by lawyers wherever they meet and discuss issues of comparative law. [...] a new approach, a new frame of mind, is perceptible ».
La mondialisation a introduit un bouleversement des mentalités et des pratiques. Les conséquences ne sont toutefois pas à surestimer. Lorsque l'on étudie l'ensemble des phénomènes connexes à cette dernière, "se découvre alors une mécanique relationnelle, complexe mais dynamique, dont les ressorts sont tout à la fois juridiques et culturels, qui stimule la circulation des modèles de droit dans l'espace mondial et favorise finalement la construction des principes et valeurs d'une justice universelle ${ }^{61}$. On retrouve ici l'idée développée par le professeur Jean Rivero lorsqu'il affirmait que « le rapprochement des jurisprudences, (est) plus important que celui des structures juridiques $»^{62}$. Cette remarque formulée à propos du droit administratif français, pour lequel la création jurisprudentielle a joué un rôle central, n'y est cependant pas limitée.

C'est donc un fait, "More and more courts, particularly within the common law world, are looking to the judgments of other jurisdictions, particularly when making decisions on human rights issues ${ }^{63}$, à l'image du célèbre arrêt de la Cour constitutionnelle sud-africaine relatif à la peine de mort, The State v. T Makwanyane ${ }^{64}$, dans lequel la Cour citait des jurisprudences de Tanzanie, d'Allemagne, des Bermudes, du Royaume-Uni, du Canada, d'Inde et des Etats-Unis. Ce phénomène de circulation des solutions juridiques ${ }^{65}$, est également observable, à des degrés divers, dans de nombreux autres systèmes juridiques ${ }^{66}$.

Ce phénomène est identifié par plusieurs universitaires sous des vocables différents, tandis qu'aux Etats-Unis, Sujit Choudhry parle

61. G. CANIVET, « Les influences croisées entre juridictions nationales et internationales. Eloge de la "bénévolance" des juges », Rev. Sc. Crim., 2005, p. 799.

62. J. RIVERO, "Vers un droit commun européen : nouvelles perspectives en droit administratif ", in Nouvelles perspectives d'un droit commun de l'Europe, M. CAPPELLETTI, (dir.), Bruxelles, Bruylant, 1978, p. 402.

63. C. L'HEUREUX-DUBÉ, " The Importance of Dialogue : Globalisation and International Impact of the Rehnquist Court », Tulsa L. J., vol. 34, 1998, p. 16.

64. CCT 3/1994, The State v. T Makwanyane and M. Mchunu, 6 juin 1995.

65. A. LE QUINIO, Recherche sur la circulation des solutions juridiques : le recours au droit comparé par les juridictions constitutionnelles, préface de Guy Canivet, Paris - ClermontFerrand, L.G.D.J., Fondation Varenne, vol. 53, 2011, 522 p. 
de « migration des idées constitutionnelles ${ }^{67}$, en France, les recherches se développent autour du recours à « l'argument de droit comparé " ${ }^{68}$. De manière générale, ces réflexions portent essentiellement sur le débat relatif aux influences réciproques entre les juridictions ressortissant de systèmes juridiques différents et sur les citations par ses dernières de droits étrangers.

Ce phénomène, qui a connu une forte croissance ses dernières années a été à l'origine d'âpres débats doctrinaux depuis les arrêts Atkins ${ }^{69}$, Lawrence v. Texas ${ }^{70}$ et Roper v. Simmons ${ }^{71}$ de la Cour suprême des Etats-Unis. Et pourtant, à quelques exceptions près, le juge constitutionnel n'est pas tenu de s'inspirer des décisions de ses homologues ${ }^{72}$. La soumission du juge constitutionnel à la circulation des solutions juridiques dans la détermination de son intime conviction se fonde sur une démarche volontariste. Néanmoins, l'intérêt d'une telle démarche, bien qu'elle ne soit pas exempte de tout reproche quant à sa légitimité ${ }^{73}$, réside dans la possibilité pour le juge de regarder comment un juge auquel incombe la même charge a pu traiter un cas équivalent. Ce type de référence, que l'on

66. U. BROBNIG, S. VAN ERP, (dir.), The Use of Comparative Law by Courts, 14ème Congrès international de droit comparé, Athènes 1997, La Haye - Londres - Boston, Kluwer Law International, 1999, 341 p. ; G. CANIVET, M. ANDENAS D. FAIRGRIEVE, (éd.), Comparative Law before the Courts, Londres, British institute of International and Comparative Law, 2004, 319 p. ; TH. DI MANNO, (dir.), Le recours au droit comparé par le juge, Bruxelles, Bruylant, coll. A la croisée des droits, 2014, 266 p. M-C. PONTHOREAU, T. GROPPI, The Use of Foreign Precedents by Constitutional Judges, Londres, Hart, coll. Hart Studies in Comparative Public Law, 2013, 452 p. L. PEROGARO, "La utilización del derecho comparado por parte de las cortes constitucionales : un análisis comparado ", Revista general de derecho público comparado, vol. 1, 2007, pp. 73-118.

67. S. CHOUDHRY (dir.), The Migration of Constitutional Ideas, Cambridge University Press, Cambridge, 2006, 448 p. Dans cet ouvrage, les contributions traitent tant la migration du point de vue des juridictions que des autres acteurs institutionnels.

68. M.-C. PONTHOREAU, « Le recours à "l'argument de droit comparé" par le juge constitutionnel. Quelques problèmes théoriques et pratiques ", in MELIN-SOUCRAMANIEN F. (dir.) L'interprétation constitutionnelle, Paris, Dalloz, coll. Thèmes et commentaires, Actes de la table ronde de l'Association internationale de droit constitutionnel organisée les 15 et 16 octobre 2004 à Bordeaux, 2005, pp. 167-184. Voir également, dans une perspective administrativiste, F. MELLERAY (dir.), L'argument de droit comparé en droit administratif français, Bruylant, coll. Droit administratif, $n^{\circ} 1$, Bruxelles, 2007, 371 p. ; G. CANIVET, "L'influence de la comparaison des droits dans l'élaboration de la jurisprudence ", in Etudes offertes au Professeur Philippe Malinvaud, Litec, Paris, 2007, pp. 133-144.

69. Atkins v. Virginia, 536 U.S. 304 (2002). retrouve essentiellement dans le domaine de la protection des droits fondamentaux, va pouvoir fournir au juge constitutionnel une aide lorsqu'il est confronté à des cas difficiles, lorsque la norme qu'il doit appliquer est insuffisamment déterminée ou lorsqu'il doit convaincre de l'acceptabilité d'une solution délicate, auquel cas, la solution étrangère pourra lui servir d'élément confortatif. Il convient de distinguer le recours à l'argument de droit comparé, dans lequel la référence ou la mention de la solution étrangère est généralement superficielle et s'inscrit dans une perspective essentiellement stratégique, du recours à la méthode juridique comparative, auquel cas la solution étrangère est susceptible de servir de motif déterminant à la prise de décision, que cela soit par son importation ou son rejet.

Néanmoins, si l'idée d'une conversation constitutionnelle est séduisante et peut parfois être identifiée, parler de " dialogue des juges » est en grande partie exagéré, voire inexact. Comme le relève Guillaume Tusseau, une telle présentation des échanges entre juridictions constitutionnelles relève d'une certaine forme d'irénisme ${ }^{74}$. Si l'inspiration par des sources étrangères n'est pas en elle-même une nouveauté, la mondialisation a entraîné une réelle intensification du phénomène. Ce constat est particulièrement prégnant devant les juridictions constitutionnelles.

En effet, ces dernières, tant pour des raisons matérielles que sur des fondements théoriques

\section{Lawrence v. Texas, 539 U.S. 558 (2003).}

71. Roper v. Simmons, 543 U.S. 551 (2005).

72. L'exemple le plus fréquemment cité est celui de la Constitution sud-africaine qui en offre la possibilité. La Section 39 de la Constitution sud-africaine de 1996 dispose en effet que : « (1) Lors de l'interprétation de la Charte des droits, (a) une cour, un tribunal ou un forum doit promouvoir les valeurs sous-jacentes d'une société ouverte et démocratique fondée sur la dignité humaine, l'égalité et la liberté; (b) doit tenir compte du droit international et ; (c) peut prendre en compte le droit étranger».

73. A. LE QUINIO, "La légitimité contrastée d'une technique juridictionnelle : le recours au droit comparé par le juge», in Le recours au droit comparé par le juge, Bruxelles, Bruylant, coll. A la croisée des droits, 2014, pp. 19-37.

74. G. TUSSEAU, "Irregulare aliquod et (tantum non) monstro simile" : remarques sur les heurs et malheurs des dialogues juridictionnels transconstitutionnels », in P. BRUNET, K. HASEGAWA, H. YAMAMOTO (dir.), Rencontres franco-japonaise autour des transferts de concepts juridiques, Paris, Mare \& Martin, Coll. Droit public, 2014, p. 110. 
ou stratégiques sont amenées à s'inspirer des solutions juridiques étrangères ${ }^{75}$. La citation de matériaux étrangers devient un élément incontournable de la politique d'une juridiction. Une Cour qui montre un intérêt marqué pour le droit comparé et s'affiche comme telle, envoi un signal fort aux autres juridictions qui participent à ce concert des juridictions ${ }^{76}$. Le Président Costa $^{77}$ par exemple, n'a jamais manqué de rappeler lorsqu'il en était le Président que la Cour européenne est à l'écoute des juridictions des Etats-parties à la Convention.

La mondialisation joue un rôle fondamental dans cette dynamique. Dans son versant universaliste, elle entraîne une réflexion plus globale dans le cadre de la protection des droits de l'homme. Certaines thématiques touchant à la protection des droits sont au cœur d'influences réciproques entre les juridictions constitutionnelles. Cette circulation des solutions juridiques est observable essentiellement dans la pratique des juridictions constitutionnelles lorsqu'elles sont chargées d'assurer la protection des droits fondamentaux. Ce contentieux spécifique se caractérise par l'existence récurrente de questionnements similaires.

C'est notamment le cas pour les législations relatives aux relations intimes entre personnes de même sexe. Plusieurs juridictions constitutionnelles ont été confrontées à ce sujet, politiquement et socialement sensible. Ces quinze dernières années, la Cour constitutionnelle d'Afrique du Sud ${ }^{78}$, la Cour suprême des EtatsUnis $^{79}$ et la Cour suprême indienne ${ }^{80}$ ont été saisies de cette question. Dans chacun de ces cas, la réflexion ou l'argumentation ont été

75. A. LE QUINIO, Recherche sur la circulation des solutions juridiques : le recours au droit comparé par les juridictions constitutionnelles, préface de Guy Canivet, L.G.D.J., Fondation Varenne, vol. 53, 2011, 522 p.

76. B. GENEVOIS, " Le Conseil d'Etat et l'ordre juridique communautaire ", E.D.C.E., vol. 31, 1980, p. 73 : "le refus du dialogue, loin d'être un signe de force, serait au contraire un aveu de faiblesse ».

77. "Questions à Jean-Paul Costa, président de la Cour européenne des droit de l'homme », A.J.D.A., 2007, p. 60.

78. CCT 11/98, National Coalition for Gay and Lesbian Equality v. Minister of Justice (the sodomy case), 9 oct. 1998.

79. Lawrence v. Texas, 539 U.S. 558 (2003).

80. Naz Foundation v. Government of NCT of Delhi, 160 DLT 277 (2009). éclairées par la comparaison des droits. Pour la dernière de ces décisions particulièrement, les juges ont mené leur réflexion dans une dynamique d'ouverture aux droits étrangers. Indépendamment de la prise en considération du cadre globalisé de la réflexion relative aux droits de la personne, les membres de la Cour sont allés très loin dans la démarche comparative, faisant notamment référence à des jurisprudences fidjienne et népalaise, pourtant très largement ignorées par ailleurs.

Le phénomène est encore plus prégnant concernant la question des droits civiques accordés aux personnes faisant l'objet d'une peine d'emprisonnement.

En effet, la question du droit de vote des détenus a ceci de particulier qu'elle a été posée successivement et de manière assez rapprochée à plusieurs juridictions constitutionnelles et suprêmes ces vingt-cinq dernières années. La première à avoir été confrontée à cette question a été la Cour suprême du Canada en 1993. Dans l'arrêt Sauvé ( $\left.n^{\circ} 1\right)$ c. Canada ${ }^{81}$, les juges devaient contrôler la constitutionnalité de la loi électorale de 1985 qui prévoyait la privation du droit de vote pour les détenus quelle que soit la gravité de l'infraction commise et la durée de la peine ${ }^{82}$. La Cour a affirmé que le caractère général et absolu de la disposition incriminée violait l'article $3^{83}$ de la Charte canadienne des droits et libertés en étant disproportionnée.

81. CSC, Sauvé v. Canada (Attorney General) (Sauvé $n^{\circ} 1$ ), [1993] 2 S.C.R. 438.

82. Dans la présente décision, le juge Arbour présentait de manière très claire les termes du débat: «la lente progression des démocraties occidentales vers le suffrage universel a franchi un point de non-retour au Canada en 1982 avec l'entrée en vigueur de l'art. 3 de la Charte. Je doute que qui que ce soit puisse maintenant être privé du droit de vote pour le motif, non simplement symbolique, mais dont la preuve a été réellement faite, que cette personne n'est pas honnête ou responsable. Au moment de l'entrée en vigueur de la Charte, les exclusions applicables au droit de vote étaient si peu nombreuses dans notre pays qu'on peut supposer à juste titre que nous avions abandonné l'idée que l'électorat doit se limiter aux " citoyens honnêtes et responsables », définis auparavant par des caractéristiques comme le fait d'être propriétaire de biens-fonds ou le sexe, en faveur d'un électorat pluraliste, qui peut très bien inclure des Canadiens qui sont des ennemis de l'État $»$.

83. Charte canadienne des droits et libertés, art. $3:$ "Tout citoyen canadien a le droit de vote et est éligible aux élections législatives fédérales ou provinciales ». 
La Cour constitutionnelle d'Afrique du Sud a examiné une question similaire dans l'arrêt August du 1er avril 199984. La Cour a enjoint la Commission électorale de prendre toutes les mesures nécessaires pour permettre aux détenus d'exercer leur droit de manière effective malgré leur incarcération, ce qu'elle n'avait pas fait ${ }^{85}$, tout en rappelant que la suppression du droit de vote pour certaines catégories de prisonniers était communément admise dans de nombreux pays. En l'espèce, le juge Sachs, au nom de la majorité, a fait référence à la jurisprudence de la Cour suprême du Canada pour rappeler que le droit de vote est fondamental dans une démocratie et qu'il doit toujours être interprété dans le sens d'une garantie plutôt que d'une privation $^{86}$. Ces références aux matériaux étrangers étaient opérées dans la perspective générale de l'importance du droit de vote accordé aux citoyens. C'est surtout l'arrêt O'Brien v Skinner ${ }^{87}$ de la Cour suprême des Etats-Unis qui oriente la décision des juges quant à la garantie de l'exercice effectif du droit et à la compétence législative en la matière ${ }^{88}$.

Puis, en $2002^{89}$, la Cour suprême du Canada a de nouveau été confrontée au contrôle de l'article 51 de la loi électorale de $1985^{90}$ en vertu de l'article 3 de la Charte. La Cour a dû effectuer un contrôle de proportionnalité approfondi entre les objectifs du Parlement et la garantie d'une liberté fondamentale car « la restriction d'un droit doit se fonder sur un motif valide du point de vue constitutionnel $»^{91}$.

84. CCT 8/99, August v. Independant Electoral Commission, 1999 (3) SA 1, \& 16: " This clearly imposes an affirmative obligation on the Commission to take reasonable steps to ensure that eligible voters are registered "; $\$ 22:$ : The Commission accordingly has not complied with its obligation to take reasonable steps to create the opportunity to enable eligible prisoners to register and vote »; $\$ 38$ : "The Commission must therefore make the necessary arrangements to enable them to vote ».

85. CCT 8/99, August v. Independant Electoral Commission, 1999 (3) SA 1, \& 57.

86. Idem, note 18 : le juge cite un extrait de la jurisprudence CSC, Haig v Canada ([1993] 2 R.C.S. 995) de la Cour suprême et l'arrêt Sauvé de la Cour d'appel de l'Ontario.

\section{O'Brien v Skinner, 414 U.S. 524 (1973).}

88. La suppression du droit de votene peutêtre automatiquement consécutive à l'incarcération mais doit être encadrée par la loi.

89. CSC, Sauvé v. Canada (Chief Electoral Officer) (Sauvé $n^{\circ} 2$ ), [2002] 3 S.C.R. 519.

90. Après l'arrêt Sauvé I, le Parlement l'avait modifié pour interdire le droit de vote aux détenus purgeant une peine d'une durée supérieure à deux ans.
La Cour a estimé, à la majorité de cinq voix contre quatre ${ }^{92}$, que la privation du droit de vote avait pour conséquence $\mathrm{d}^{\prime}$ '« abandonner un important moyen de leur inculquer (aux détenus) des valeurs démocratiques et le sens des responsabilités sociales $\nu^{93}$. Les juges de la majorité ont notamment invoqué la décision August de la Cour suprême sud-africaine, qui rattachait l'exercice de la citoyenneté à la dignité humaine ${ }^{94}$ et ont considéré que le gouvernement « n’a pas réussi à établir un lien rationnel entre la privation du droit de vote [...] et les objectifs d'accroître le respect de la règle de droit et d'assurer l'infliction d'une peine appropriée $»^{95}$. Les juges dissidents ont, pour leur part, privilégié les décisions des juges anglais, américains, et de la Cour européenne des droits de l'homme. La majorité des matériaux étrangers invoqués dans l'opinion dissidente le sont sous un titre "L'inhabilité des prisonniers à voter dans les provinces canadiennes, dans d'autres pays et à l'échelle internationale : dosages raisonnables possibles ${ }^{96}$. Les juges ont souhaité montrer que des restrictions au droit de vote des détenus étaient acceptées aux Etats-Unis ${ }^{97}$ et dans le cadre du Conseil de l'Europe ${ }^{98}$, dans les Etats européens ${ }^{99}$ et dans les pays de common law ${ }^{100}$.

91. CSC, Sauvé v. Canada (Chief Electoral Officer) (Sauvé $\left.n^{\circ} 2\right)$, 2002, 3 S.C.R. 519, § 20 : en l'espèce, l'opinion majoritaire fait référence à un principe de droit allemand et à un article du juge Dieter Grimm.

92. Les juges dissidents ne s'opposent pas en soi sur la question du droit de vote, mais sur la question de la retenue judiciaire. Le juge Gonthier l'explique : " Nos différences résident principalement dans le fait que le Juge en chef souscrit à une philosophie selon laquelle le retrait temporaire du droit de vote aux criminels va à l'encontre de la règle de droit, de la démocratie et du droit de vote, alors que je préfère la retenue judiciaire à l'égard de l'opinion raisonnable du législateur, selon laquelle cette mesure renforce ces mêmes caractéristiques de la société canadienne ", Sauvé v. Canada (Chief Electoral Officer) (Sauvé $n^{\circ}$ 2), 2002, 3 S.C.R. 519, \& 68.

93. CSC, Sauvé v. Canada (Chief Electoral Officer) (Sauvé $\left.n^{\circ} 2\right)$, [2002] 3 S.C.R. 519, § 38.

94. CCT 8/99, August v. Independant Electoral Commission, 1999 (3) SA 1, \& 17 : "The vote of each and every citizen is a badge of dignity and of personhood ».

95. CSC, Sauvé v. Canada (Chief Electoral Officer) (Sauvé $n^{\circ}$ 2) [2002] 3 S.C.R. 519, § 53.

96. Ibidem, $\S \S 122-134$.

97. Le panorama dressé par le juge Gonthier montre qu'une majorité des Etats suppriment le droit de vote pour les personnes condamnées pour actes criminels graves. Cette suppression peut perdurer pendant la libération conditionnelle, voire être définitive, ce que la Cour suprême a estimé constitutionnel (Richardson c. Ramirez, 418 U.S. 24 (1974)). 
Lorsqu'elle a été saisie en 2004, dans l'affaire Minister of Home Affairs v. NICRO ${ }^{101}$, la Cour constitutionnelle d'Afrique du Sud a dû examiner la conformité à la Constitution de la loi électorale qui prévoyait la suppression du droit de vote pour les détenus condamnés à une peine de prison ferme en vertu du Electoral Laws Amendment Act promulgué en novembre 2003. La Cour s'est penchée, au même titre que son homologue canadien, sur la conciliation entre l'exercice d'une liberté fondamentale et les restrictions invoquées par le Ministre des affaires intérieures et sur la question des contraintes logistiques entraînée en cas de vote des détenus.

Dans cette affaire, les membres de la Cour constitutionnelle sud-africaine ont analysé de manière approfondie la jurisprudence de la Cour suprême du Canada. Le juge Chaskalson explique que l'arrêt Sauvé est topique de cette problématique et en rappelle précisément les faits $^{102}$ et les apports essentiels.

98. Comm. eur. dr. h., 4 juillet 1983, H. c. Pays-Bas, requête no 9914/82, D.R. 33, p. 24s : "De telles limitations s'expliquent par l'idée que certaines condamnations marquent d'infamie pour un temps déterminé qui peut être pris en considération par la législation quant à l'exercice des droits politiques. Bien qu'à première vue il puisse paraître rigide qu'une condamnation supérieure à un an ait toujours pour conséquence une suspension de l'exercice du droit de vote pour trois ans, la Commission ne trouve pas qu'une telle mesure excède les limitations justifiables dans le cadre de l'article 3 du Protocole additionnel ».

99. Parmi ces exemples, le juge Gonthier se réfère à deux reprises à l'arrêt Pearson c. Secretary of State for the Home Department ([2001] E.W.J. No. 1566 (QL)) de la Divisional Court (aux \& 108 \& 132) dans lequel les juges britanniques étaient confrontés à la question de la compatibilité du Representation of the People Act de 1983 qui déchoit les détenus de participer à certaines élections au Human Rights Act de 1998. La Cour jugea conforme les dispositions de la loi à la Convention, le juge Kennedy affirmant que "Comme il appartient au Parlement de décider des conséquences d'une déclaration de culpabilité en établissant les attributions et les obligations du tribunal (ou autre organisme) qui inflige la peine, il s'ensuit nécessairement que la ligne de démarcation doitêtre tracée quelque partet que, lors d'un examen ultérieur, on pourra soutenir que la ligne aurait dû être tracée ailleurs. Cependant, par respect pour le législateur, les tribunaux ne doivent pas se laisser facilement convaincre de condamner une mesure législative, surtout lorsqu'elle a été adoptée dans le cadre d'une loi principale à l'issue d'une évaluation minutieuse et dans le contexte de l'inquiétude croissante de la population face à la criminalité ». Il faut noter que dans son raisonnement sur cette affaire, le juge Kennedy a fait plusieurs références à l'arrêt Sauvé $\left(n^{\circ} 1\right)$ ainsi qu'à la décision de la Cour d'appel fédérale en l'affaire Sauvé ( $n^{\circ}$ 2) qui a été ultérieurement annulée par la Cour suprême du Canada.

100. Dans la majorité des Etats européens, les détenus ne sont pas déchus de leurs droits civiques tandis qu'en Australie, en Nouvelle-Zélande et au Royaume-Uni, les détenus condamnés à des peines lourdes le sont.
En 2005, ce fut au tour de la Cour européenne des droits de l'homme d'examiner la requête de monsieur Hirst, qui se plaignait d'avoir été frappé, en raison de sa condition détenu condamné purgeant sa peine, d'une privation totale du droit de vote ; il invoquait l'article $3 \mathrm{du}$ protocole $n^{\circ} 1$ à la Convention ${ }^{103}$, pris isolément et combiné avec l'article 14, ainsi que l'art. 10 de la Convention. Si la Cour retient l'idée d'une marge nationale d'appréciation en la matière, elle rappelle que les limitations apportées à l'exercice de ces droits doivent être proportionnées aux objectifs poursuivis et doivent être justifiées par un but légitime ${ }^{104}$. Dans la partie « en fait » de l'arrêt, les juges, après avoir exposé les grands textes internationaux en la matière et la pratique dans les Etats contractants ${ }^{105}$, ont analysé la « jurisprudence pertinente d'autres Etats ${ }^{106}$. Les juges ont détaillé successivement et de manière précise les raisonnements établis dans les arrêts Sauvé $\left(\mathrm{n}^{\circ} 1 \text { et } 2\right)^{107}$ et August ${ }^{108}$.

En 2007, la même question s'est posée devant la High Court australienne dans l'affaire Roach ${ }^{109}$. En l'espèce, la Cour était saisie de l'examen de la constitutionnalité des amendements au Commonwealth Electoral Act de 1918 issus de la section 93(8AA) de l'Electoral and Referendum Amendment (Electoral Integrity and

101. CCT 03/04, Minister of Home Affairs v. National Institute for Crime Prevention and the Reintegration of Offenders (NICRO) and others, 3 mars 2004.

\section{Ibidem, $\S \S 58-64$.}

103. Art. $3 d u$ Protocole $n^{\circ} 1$ additionnel à la Convention européenne de sauvegarde des droits de l'Homme et des libertés fondamentales : "Les hautes parties contractantes s'engagent à organiser, à des intervalles raisonnables, des élections libres au scrutin secret, dans des conditions qui assurent la libre expression de l'opinion du peuple sur le choix du corps législatif ».

104. C.E.D.H., 06 oct. 2005, Hirst c. Royaume-Uni ( $\left.n^{\circ} 2\right)$, aff. $n^{\circ}$ 74025/01, \& 62: "il lui faut s'assurer (à la Cour) que les limitations ne réduisent pas les droits dont il s'agit au point de les atteindre dans leur substance même et de les priver de leur effectivité, qu'elles poursuivent un but légitime et que les moyens employés ne se révèlent pas disproportionnés ».

105. Ibidem, $\S \S 33-34$.

106. Les arrêts et tendances présentés par la Cour avaient été évoqués par les requérants.

107. C.E.D.H., 06 oct. 2005, Hirst c. Royaume-Uni ( $\left.n^{\circ} 2\right)$, aff. $n^{\circ}$ 74025/01, §§ 35-37.

108. Ibidem, $\S \S 38-39$.

109. Roach v. Electoral Commissioner [2007] HCA 43. 
Other Measures) Act qui prive du droit de vote les détenus pour les élections parlementaires. Si les juges ont reconnu la contrariété de ces amendements à l'article 24 de la Constitution, ils ont néanmoins accepté la constitutionnalité des dispositions précédentes de la loi qui prévoyaient une suppression du droit de vote des détenus condamnés à une peine d'au moins trois ans.

Dans cette décision, la High Court a rappelé les principaux apports des jurisprudences Sauvé $\left(n^{\circ} 2\right)^{110}$ et Hirst ${ }^{111}$. Le juge Gleeson, dans son opinion $^{112}$, relativise l'apport de ces exemples. D'après lui, ils sont très instructifs au niveau de la motivation, les opinions minoritaires et majoritaires s'opposant essentiellement sur la marge d'appréciation que les juridictions devraient accorder au législateur pour examiner la question de la proportionnalité113. Mais il craint que la transposition du principe de proportionnalité dans le système juridique australien puisse être inappropriée et considère que les arguments de l'opinion dissidente du juge Gonthier seraient conformes à la conception australienne $\mathrm{e}^{114}$.

Il ressort de ce qu'il précède que si les hautes juridictions consultent et étudient les décisions de leurs homologues, elles ne les suivent pas nécessairement. Ainsi, la Cour suprême du Royaume-Uni s'est opposée à la Cour européenne des droits de l'homme qui avait condamné le Royaume-Uni dans la décision Hirst $\mathrm{n}^{\circ} 2$. Les juges anglais, qui considèrent que le droit de vote des prisonniers ne relève pas d'un « aspect [...] fondamental » du droit britannique ${ }^{115}$ sont seulement tenus de prendre en compte, sans nécessairement la suivre, la jurisprudence de la Cour européenne en vertu de la section $2 \mathrm{du}$ Human Rights Act. C'est d'ailleurs ce qu'avaient

110. Ibidem, $\S \S 13-15$.

111. Ibidem, $\S 16$

112. La décision a été rendue à la majorité de quatre contre deux. La majorité était composée du juge Gleeson et des juges Crennan, Gummow et Kirby qui ont rédigé une opinion concourante à celle du juge Gleeson.

113. Roach v. Electoral Commissioner [2007] HCA 43, § 17.

114. Ibidem, $\S 19$.

115. $R$ (Chester) v Secretary of State for Justice; McGeoch $v$ Lord President of the Council and another [2013] UKSC 63, Lord Mance, $\S 35$. déjà rappelé les juges de la Cour suprême du Royaume-Uni dans la décision R v. Horncastle ${ }^{116}$ en considérant que la Cour européenne avait méconnu un aspect important du système britannique et en espérant ainsi infléchir sa politique jurisprudentielle relative au droit de vote des détenus. La contrariété entre la position de la Cour européenne et celle de la Cour suprême du Royaume-Uni qui est toujours patente et a connu plusieurs étapes, a été nourri par la circulation des solutions juridiques. La Cour de Strasbourg, dans une affaire portant sur la même problématique mais concernant cette fois l'Italie, Scoppola c. Italie $\left(n^{\circ} 3\right)^{117}$, a confirmé, en la précisant, sa politique jurisprudentielle entamée avec la décision Hirst tout en se référant à des éléments de droit comparé et aux décisions Sauvé, August et Roach.

Cette circulation des solutions juridiques peut également affecter des problématiques différentes de manière étonnante. Récemment, la Cour constitutionnelle de la Fédération de Russie, dans une décision du 14 juillet 2015, s'est soustraite à ses obligations conventionnelles en affirmant que la Constitution russe primait toujours sur la Convention européenne des droits de l'Homme. En effet, la Cour russe a déclaré dans cette décision que « dans l'hypothèse où des principes et des normes de la Constitution de la Fédération russe sont illégalement mis en cause par une jurisprudence de la Cour européenne y compris lorsqu'elle s'adresse aux Etats en se fondant sur l'interprétation des dispositions de la CEDH, la Russie peut exceptionnellement se retirer des obligations qui lui sont imposées lorsqu'une telle dérogation est la seule voie possible pour éviter les violations des principes et normes fondamentaux de la Constitution de la Fédération de Russie ${ }^{118}$. Il est intéressant de constater que dans sa décision, la Cour constitutionnelle russe fonde sa position sur le respect des dispositions de la Convention de

\section{6. $R$ v Horncastle [2009] UKSC 14.}

117. CEDH, G.C. 23 mai 2012, Scoppola c. Italie ( $\left.n^{\circ} 3\right)$.

118. \$2.2. La décision a été publiée le 27 juillet 2015, dans la Gazette russe, numéro fédéral $n^{\circ} 6734$ (в « Российской Газете ", Федеральный выпуск, $n^{\circ}$ 6734) disponible sur le site Internet de la Cour constitutionnelle russe (http://doc.ksrf.ru/ decision/KSRFDecision201896.pdf). L'auteur de la présente contribution remercie Victoria Chiu, Maître de conférences à l'Université Lyon III, pour sa traduction de la décision. 
Vienne du 23 mai 1969 sur le droit des traités ${ }^{119}$ en considérant que la protection offerte par le système juridique russe est plus complète que celle garantie par la CEDH.

En outre, si la Cour reconnaît la dimension exceptionnelle du refus par les Etats parties de suivre l'interprétation et l'application de la CEDH, elle mentionne les prises de position des Cours constitutionnelles allemande, italienne et autrichienne qui ont invoqué l'impossibilité de réduire le niveau de protection des droits fondamentaux tels qu'ils sont garantis par le droit interne pour s'opposer au respect de certaines de leurs obligations conventionnelles ${ }^{120}$. La Cour constitutionnelle termine cette analyse de droit comparé avec la décision de la Cour suprême du Royaume-Uni qui avait rejeté l'application inconditionnelle des décisions de la Cour européenne des droits de l'Homme à propos des droits électoraux des détenus ${ }^{121}$. La Cour constitutionnelle russe rejette donc la solution de la Cour européenne et le principe même de l'autorité de ses décisions en se fondant notamment sur des solutions d'autres juridictions constitutionnelles européennes. La Cour russe se sert donc du " précédent étranger » pour écarter le « précédent international » alors même qu'il bénéficie d'une force normative inférieure.

L'intérêt de ces jurisprudences, au-delà de la question de la garantie des droits civiques des détenus, réside dans l'influence de ces référencements croisés et dans le fait que ces différentes juridictions empruntent

119. Notamment l'article 26 sur l'interprétation de bonne foi des textes internationaux ainsi que sur l'article 46 permettant aux Etats de bloquer l'action de certaines dispositions d'un traité international.

120. Voir \$4, alinéas 5, 6, 7 et 8 de la décision. En effet, la Cour constitutionnelle russe mentionne deux décisions de ses homologues allemands : celle du 29 mai 1974 dans l'affaire 2 BvL 52/71 (BVerfGE 37, 271) [«Solange-I»] et celle du 14 octobre 2004 dans l'affaire 2BvR 1481-1404 (BVerfGE 111, 307). En outre, elle mentionne les arrêts suivants de la Cour constitutionnelle italienne: ceux du 19 novembre 2012, $n^{\circ}$ 264/2012 et du 22 Octobre 2014, $n^{\circ}$ 238/2014.

121. R. (Chester) v. Secretary of State for Justice; McGeoch v The Lord President of the Council \& Anor, [2013] UKSC 63.

122. E. PICARD, «L'état du droit comparé en France», R.I.D.C., $n^{\circ}$ 4, 1999, p. 886. L'auteur affirme que le droit comparé n'est plus en France " un objet de simple curiosité intellectuelle, réservé à quelques esprits jugés à l'époque quelque peu marginaux en ce qu'ils se piquaient d'un goût bizarre pour ce que l'on considérait encore comme une sorte d'exotisme juridique ». successivement une partie de la substance des jurisprudences étrangères antérieures, montrant ainsi comment le recours au droit comparé est encouragé par la mondialisation. Le droit comparé est donc devenu un outil incontournable de la pensée et de la dynamique juridique, entraînant également un changement du statut de la discipline ${ }^{122}$.

\section{Perspectives et conclusion}

Le recours au droit comparé par une juridiction montre une certaine déférence à l'égard du droit étranger, invitant ainsi les autres à faire de même afin de bénéficier de la fécondité de la transmission des idées juridiques entre les peuples ${ }^{123}$. Les juges ont également pris conscience qu'ils sont observés par leurs homologues étrangers, entrainant une concurrence latente qui les incite à " une sorte de surenchère du "plus civilisé" et du "plus protecteur" »124. C'est la jurisprudence qui donne l'image de la juridiction. Immanquablement, lorsque les juges étudient les décisions étrangères ou en entendent parler dans les medias, ils finissent par se comparer à leurs homologues.

Le temps est révolu où un ou deux systèmes juridiques servaient de phares à l'ensemble des autres. Au contraire, dans l'exemple choisi, le modèle américain en général et la jurisprudence de la Cour suprême des Etats-Unis en particulier ont davantage été invoqués comme des cas extrêmes de ce qui était acceptable dans un Etat démocratique.

Le regard des juges constitutionnels est aujourd'hui largement tourné vers l'extérieur et propice au comparatisme juridique. Nous avons changé d'ère juridique pour pénétrer dans une forme de " cosmopolitisme constitutionnel ». Un véritable revirement a été opéré. Là où les

123. G. DEL VECCHIO, "La communicabilité du droit et les doctrines de G.-B. Vico », in Introduction à l'étude du droit comparé - Recueil d'études en l'honneur d'Edouard Lambert, t.2, Paris, Sirey - L.G.D.J., 1938, p. 591.

124. L. HENNEBEL, "Les amici curiae : acteurs de la "convergence" des droits de l'homme ", in ALLARD J., HAARSCHER G., HENNEBEL L., LEWKOWICZ G., Juger les droits de l'homme - Europe et Etats-Unis face-à-face, Bruxelles, Bruylant, coll. Penser le droit, $n^{\circ} 10,2008$, p. 91. 
migrations juridiques se faisaient essentiellement dans une direction, aujourd'hui les variables juridiques circulent ; « the process of international influences has changed from reception to dialogue. Judges no longer simply receive the cases of other jurisdictions and then apply them or modify them for their own jurisdiction ${ }^{125}$. Pour les juges, cette circulation concerne essentiellement les solutions juridiques. En effet, lorsqu'ils font référence à des décisions étrangères, ce sont des exemples de solutions à des problèmes concrets qu'ils cherchent. Pour eux, l'objectif n'est pas la greffe d'une règle de droit, mais bien la réception d'un raisonnement.

Le temps est venu d'ouvrir son esprit aux solutions externes. Une institution, une juridiction, particulièrement lorsqu'elle est chargée du contrôle de constitutionnalité des lois, ne peut plus être totalement repliée sur elle-même. Il est de l'intérêt de tous - les avocats et les justiciables l'ont bien compris - d'étudier les pratiques juridiques des autres pays afin d'observer la façon dont ils réagissent lorsqu'ils sont confrontés à des problèmes similaires. Pour le Président Canivet, « qu'elle soit interne ou internationale, une juridiction ne peut être un lieu clos, c'est au contraire un carrefour, un carrefour circulaire où se croisent de manière ordonnée les décisions de justice qui construisent un droit en devenir permanent » .

Les acteurs juridiques, quels qu'ils soient, lorsqu'ils doivent se pencher sur les questions qui se présentent à eux, doivent s'interroger dans le cadre d'un environnement global qui a muté, s'apparentant davantage à un espace mondial. Le développement du phénomène de globalisation consécutif à l'essor des moyens de communication a obligé les juristes à s'émanciper de leur «bulle nationale ». Ces derniers doivent faire face à des modèles juridiques disparates, eux-mêmes le plus souvent imbriqués dans des aires de protection des droits supranationales qui influent directement sur le contenu matériel des ordres juridiques nationaux.

La mondialisation, si elle entraîne des craintes, suscite également des espoirs ${ }^{127}$. Il faut espérer, avec Justice Breyer qu'elle « conduira finalement à un système de droit qui servira mieux les intérêts de ceux qui doivent en principe en bénéficier, et qui sont justement les citoyens ordinaires de nos pays $»^{128}$.

125. C. L'HEUREUX-DUBÉ, "The Importance of Dialogue : Globalisation and International Impact of the Rehnquist Court », Tulsa L. J., vol. 34, 1998, p. 17.

126. G. CANIVET, "Les influences croisées entre juridictions nationales et internationales. Eloge de la "bénévolance" des juges », Rev. Sc. Crim., 2005, p. 817.

127. Sur les perceptions divergentes de la mondialisation, voir E. FOUGIER, "Perceptions de la mondialisation en France et aux Etats-Unis », Politique étrangère, $n^{\circ}$ 3, 2001, pp. 569-595.

128. S. BREYER, "Inspiration réciproque et mondialisation des flux économiques ", in Diversité des systèmes juridiques et inspiration réciproque des juges, Actes du colloque du Cercle France-Amériques du 24 oct. 2006, Cycle Attractivité économique du droit, LPA, $n^{\circ} 112,2008, p .6$. 


\section{BIBLIOGRAPHIE}

- ALLARD J., GARAPON A., Les juges dans la mondialisation. La nouvelle révolution du droit, Paris, éd. du Seuil, coll. La République des Idées, 2005, 96 p.

- ALLARD J., VAN WAYENEBERGE A., « De la bouche à l'oreille? Quelques réflexions autour du dialogue des juges et de la montée en puissance de la fonction de juger », R.I.E.J., vol. 61, 2008, pp. 109-129.

- ARNAUD A.-J., Entre modernité et mondialisation - Leçons d'histoire de la philosophie du droit et de l'Etat, Paris, L.G.D.J., coll. Droit et société, Série Droit, vol. 20, 1998, $307 \mathrm{p}$.

- BASEDOW J., « Vie universelle. Droit national ? A propos de la mondialisation du droit », in Mélanges en l'honneur de Denis Tallon - D'ici, d'ailleurs : harmonisation et dynamique du droit, Paris, SLC, 1999, pp. 223-238.

- BIRNBAUM P., « Le type d'Etat tient toujours », in L'architecture du droit, mélanges en l'honneur de Michel Troper, Paris, Economica, 2006, pp. 185-194.

- BLANC H., « De l'internationalisation des économies à leur mondialisation, le regard de l'économiste », in Images de la mondialisation - La construction sociale d'une représentation, Actes du colloque international "Représentations de la Mondialisation", tenu à Brest les 17 et 18 nov. 2005, Rennes, P.U.R., coll. Des Sociétés, 2008, pp. 39-52.

- BOMPARD-PORTE M., « Questions de mots ", in Images de la mondialisation - La construction sociale d'une représentation, Actes du colloque international " Représentations de la Mondialisation ", tenu à Brest les 17 et 18 nov. 2005, Rennes, P.U.R., coll. Des Sociétés, 2008, pp. 17-22.

- BREYER S., "Inspiration réciproque et mondialisation des flux économiques ", in Diversité des systèmes juridiques et inspiration réciproque des juges, Actes du colloque du Cercle France-Amériques du 24 oct. 2006, Cycle Attractivité économique du droit, LPA, n 112,2008 , pp. 5-6.

- BROBNIG U., VAN ERP S., (dir.), The Use of Comparative Law by Courts, 14ème Congrès international de droit comparé, Athènes 1997, La Haye - Londres - Boston, Kluwer Law International, 1999, 341 p.

- BULLIER A. J., « Le droit comparé est-il un passe temps inutile ? », R.D.I.D.C., $\mathrm{n}^{\circ} 2$ \& 3 , 2008, pp. 159-172.

- CAILlE A., DUFOIX S., (dir.), Le tournant global des sciences sociales, Paris, éd. La Découverte, 2013, 440 p.

- CANIVET G., ANDENAS D. FAIRGRIEVE M., (éd.), Comparative Law before the Courts, Londres, British institute of International and Comparative Law, 2004, 319 p.

- CANIVET G., « La convergence des systèmes juridiques par l'action du juge », in De tous horizons, Mélanges Xavier Blanc-Jouvan, Paris, S.L.C., 2005, pp. 11-24.

- CANIVET G., «Les influences croisées entre juridictions nationales et internationales. Eloge de la "bénévolance" des juges », Rev. Sc. Crim., 2005, pp. 799-817.

- CANIVET G., " L'influence de la comparaison des droits dans l'élaboration de la jurisprudence », in Etudes offertes au Professeur Philippe Malinvaud, Litec, Paris, 2007, pp. 133-144.

- ChEvallier J., « Mondialisation du droit ou droit de la mondialisation ? ", in C.-A. MORAND, (dir.), Le droit saisi par la mondialisation, Bruylant, Coll. De droit international, Ed. De l'Université de Bruxelles, Bruxelles, Helbing \& Lichtenhahn Verlag, 2001, pp. 37-61.

- CHOUdHRY S. (dir.), The Migration of Constitutional Ideas, Cambridge University Press, Cambridge, 2006, 448 p.

- COSNARD M., " La création normative des Etats - Point de vue publiciste », in La mondialisation du droit, Colloque des 13 , 
14 et 15 sept. 1999 de Dijon, LOQUIN E., KESSEDJIAN C. (dir.), Travaux du centre de recherche sur le droit des marchés et des investissements internationaux, vol. 19, Paris, Litec, 2000, p. 149-172.

- DELMAS-MARTY M., Trois défis pour un droit mondial, Paris, éd. du Seuil, coll. Essais, 1998, $201 \mathrm{p}$.

- DELMAS-MARTY M., Critique de l'intégration normative, Paris, PUF, coll. Les voies du droit, 2004, $330 \mathrm{p}$.

- DELMAS-MARTY M., « Mondialisation et montée en puissance des juges ", in Le dialogue des juges, Actes du colloque organisé le 28 avril 2006 à l'Université libre de Bruxelles, Bruxelles, Bruylant, coll. Les Cahiers de l'Institut d'études sur la Justice, vol. 9, 2007, pp. 94-114.

- DELMAS-MARTY M., " Les processus de mondialisation du droit », in MORAND C.-A., (dir.), Le droit saisi par la mondialisation, Bruxelles, Bruylant, Editions de l'Université de Bruxelles, Collection de droit international, $n^{\circ} 46$, Helbing \& Lichtenhahn Verlag, 2001, pp. 63-80.

- DE LAPRADELLE G., «Juridicisation de lasociété et globalisation », in CHEMILLIER-GENDREAU M., MOULIER-BOUTANG Y., (dir.), Le droit dans la mondialisation - Une perspective critique, Paris, P.U.F., 2001, pp. 21-26.

- DEL VECCHIO G., " La communicabilité du droit et les doctrines de G.-B. Vico », in Introduction à l'étude du droit comparé Recueil d'études en l'honneur d'Edouard Lambert, t.2, Paris, Sirey - L.G.D.J., 1938, pp. 591601.

- ERRERA R., "The Use of Comparative Law Before the French Administrative Law Courts ", in CANIVET G., ANDENAS M., FAIRGRIEVE D. (éd.), Comparative Law before the Courts, British institute of International and Comparative Law, Londres, 2004, pp. 153-163.

- GERBER D. J., "Globalization and Legal Knowledge : Implications for Comparative Law», Tul. L. Rev., n 75, 2001, pp. 949-975.
- GLENN P. H., « La tradition juridique nationale », R.I.D.C., vol. 55, n ${ }^{\circ}$, pp. 263-278.

- HASSENTEUFEL P., « De la comparaison internationale à la comparaison transnationale», R.F.S.P., 2005, pp. 113-132.

- JACQUET P.,SACHWALD P., «Mondialisation : la vraie rupture du 20ème siècle », Politique étrangère, $n^{\circ}$ 3-4, 2000, pp. 597-612.

- KOH H. H., « The Globalization of Freedom », Yale J. Int'l L., vol. 26, 2001, pp. 305-312.

- KUTTNER R. L., " Development, Globalization, and Law », Mich. J. Int'l L., ${ }^{\circ}$ 26, 2004, pp. 19-38.

- LE QUINIO A., Recherche sur la circulation des solutions juridiques : le recours au droit comparé par les juridictions constitutionnelles, préface de Guy Canivet, Paris - Clermont-Ferrand, L.G.D.J., Fondation Varenne, vol. 53, 2011, 522 p.

- LE QUINIO A., (dir.), Les réactions constitutionnelles à la globalisation, Bruxelles, Bruylant, coll. A la croisée des droits, 2016, $351 \mathrm{p}$.

- LE QUINIO A., "La légitimité contrastée d'une technique juridictionnelle : le recours au droit comparé par le juge », in Le recours au droit comparé par le juge, Bruxelles, Bruylant, coll. A la croisée des droits, 2014, pp. 19-37.

- L'HEUREUX-DUBÉ C., « The Importance of Dialogue : Globalisation and International Impact of the Rehnquist Court », Tulsa L. J., vol. 34, 1998, pp. 15-40.

- MICHALET C.-A., « Les métamorphoses de la mondialisation, une approché économique ", in La mondialisation du droit, Colloque des 13, 14 et 15 sept. 1999 de Dijon, LOQUIN E., KESSEDJIAN C. (dir.), Travaux du centre de recherche sur le droit des marchés et des investissements internationaux, vol. 19, Paris, Litec, 2000, pp. 11-42.

- MOCKLE D., « Mondialisation, droit des peuples et Etat de droit ", in MOCKLE D. (dir.), Mondialisation et Etat de droit, Bruxelles, Bruylant, coll. Mondialisation et droit international, 2002, pp. 1-23. 
- OST F., KERCHOVE M. (van de), Le droit ou les paradoxes du jeu, coll. Les voies du droit, Paris, P.U.F, 1992, 268 p.

- OST F. et KERCHOVE M. (van de), « Le juge entre ordre et désordre ", in Dire le droit, faire justice, coll. Penser le droit, Bruxelles, Bruylant, 2007, pp. 61-77.

- PICARD E., «L'état du droit comparé en France », R.I.D.C., nº 4, 1999, pp. 885-915.

- PONTHOREAU M.-C., GROPPI T., The Use of Foreign Precedents by Constitutional Judges, Londres, Hart, coll. Hart Studies in Comparative Public Law, 2013, 452 p.

- PONTHOREAU M.-C., « Le recours à "l’argument de droit comparé" par le juge constitutionnel. Quelques problèmes théoriques et pratiques », in MELINSOUCRAMANIEN F. (dir.), L'interprétation constitutionnelle, Paris, Dalloz, coll. Thèmes et commentaires, Actes de la table ronde de l'Association internationale de droit constitutionnel organisée les 15 et 16 octobre 2004 à Bordeaux, 2005, pp. 167-184.

- PONTHOREAU M.-C., « La concurrence entre Common Law et droit civil. Existet-il véritablement une concurrence entre Common Law et tradition civiliste ? Le point de vue du comparatiste de droit public », in DU BOIS DE GAUDUSSON J., FERRAND F. (dir.), La concurrence des systèmes juridiques, Actes du colloque organisé le 20 octobre 2006 à l'Université Jean Moulin Lyon III, Aixen-Provence, P.U.A.M., 2008, pp. 35-47.

- RIVERO J., " Droit administratif français et droits administratifs étrangers », in DE LAUBADERE A., MATHIOT A., RIVERO J., VEDEL G., Pages de doctrine, Paris, L.G.D.J., 1980 , t. 2, pp. 475-485.

- RIVERO J., « Vers un droit commun européen : nouvelles perspectives en droit administratif », in Nouvelles perspectives d'un droit commun de l'Europe, M. CAPPELLETTI, (dir.), Bruxelles, Bruylant, 1978, pp. 389-406.
- SUEUR J.-J., « Droit constitutionnel global ou droit global des constitutions ? Eléments d'analyse », in A. LE QUINIO, (dir.), Les réactions constitutionnelles à la globalisation, Bruxelles, Bruylant, coll. A la croisée des droits, 2016, pp. 17-52.

- TURPIN D., « Mondialisation et normes juridiques (Pour un nouveau contrat social global) », in L'esprit des institutions, l'équilibre des pouvoirs. Mélanges en l'honneur de Pierre Pactet, Paris, Dalloz, 2003, pp. 439-449.

- TUSSEAU G., « Un chaos conceptuel qui fait sens : la rhétorique du constitutionnalisme global », in J.-Y. CHEROT, B. FRYDMAN (dir.), La science du droit dans la globalisation Bruxelles, Bruylant, 2012, pp. 182-227.

- TUSSEAU G., " "Irregulare aliquod et (tantum non) monstro simile" : remarques sur les heurs et malheurs des dialogues juridictionnels transconstitutionnels », in P. BRUNET, K. HASEGAWA, H. YAMAMOTO (dir.), Rencontres franco-japonaise autour des transferts de concepts juridiques, Paris, Mare \& Martin, Coll. Droit public, 2014, pp. 97-140.

- TWINING W., Globalization and Legal Theory, Evanston, Northwestern University Press, 2000, 279 p.

- TWINING W., « Diffusion and Globalization Discourse », Harv. Int'l L. J., vol. 47, 2006, pp. 507-514.

- VAN HOUTTE H., « La modélisation substantielle », in LOQUIN E., KESSEDJIAN C. (dir.), La mondialisation du droit, Colloque des 13, 14 et 15 sept. 1999 de Dijon, Litec, Travaux du centre de recherche sur le droit des marchés et des investissements internationaux, vol. 19, 2000, pp. 207-236.

- WESTBROOK D. A., " Keynote Adress : Theorizing the Diffusion of Law : Conceptual Difficulties, Unstable Imaginations, and the Effort to Think Gracefully Nonetheless ", Harv. Int'l L. J., vol. 47, 2006, pp. 489-505. 